\title{
Vote-Based Wind Speed Forecast Scheme Built Upon Different Artificial Neural Network Models
}

\author{
Junghoon Lee, Gyung-Leen Park* \\ Dept. of Computer Science and Statistics, \\ Jeju National University \\ Republic of Korea \\ \{jhlee,glpark\}@jejunu.ac.kr
}

\begin{abstract}
This paper designs and develops a wind speed forecast model based on artificial neural networks and voting strategies, aiming at efficiently integrating renewable energies into the power network. The hour-by-hour speed records collected in Jeju city during the past 15 years are classified and converted to create sequential, monthly, and seasonal forecast models, respectively. To predict the next hour speed, the speed records of the previous 5 hours are simultaneously fed to each model first. Then, the voting process picks and averages the two predictions having the best proximity out of 3 . The evaluation procedure compares the predicted values and the actual speeds of 2014, which have not been used for training, and finds out that the maximum daily root mean square error for the proposed scheme is smaller than other stand-alone methods by 0.11 mps. Moreover, the vote-based scheme avoids the worst case mis-prediction.
\end{abstract}

Keywords: smart grid, wind power generation, wind speed prediction, artificial neural network, voting

\section{Introduction}

In modern power systems, which pursue energy efficiency and eco-friendliness, renewable energies are drawing more and more attention [1]. By means of the integration of electricity created from such energies, we can avoid building power plants which burn fossil fuels or invoke nuclear reactions. Many areas or countries usually have the potential of their own renewable energy sources such as wind, sunlight, wave, and the like. However, such energies cannot be easily integrated into the main grid due to their intermittent nature [2]. For example, the electricity can be over-generated beyond the demand and abandoned when it is windy. In addition, no electricity can be generated even when the demand is high and the main grid must meet the demand solely. This problem arises from the fact that electricity cannot be economically stored for later use [3]. For more efficient operation, the main grid must decide how much energy it will generate from its power generation facility, taking into account the amount of electricity that can come from renewable energies.

To this end, it is necessary to forecast the availability of renewable sources as accurately as possible. For the case of wind energy, it is necessary to forecast the wind speed. Actually, the wind speed forecast has long been a problem challenged by many researchers and engineers from many countries $[4,5]$. Not to mention, there is no common method for the wind speed prediction, but each area has its own model. Moreover, the challenges have tried a variety of techniques such as ANNS (Artificial Neural Networks), fuzzy logic, ARIMA (Auto Regressive Integrated Moving Averate), and others, and no sole method outperforms others. They are usually combined for better

${ }^{*}$ Prof. Gyung-Leen Park is the corresponding author. 
accuracy with different weight on different models [6]. Here, ANNs are most widely used technique for modeling and forecasting the complex and nonlinear behavior of target objects [7]. It finds the dependency between input and output variables through the training phase, while the training data sets are generally obtained by a series of observations or measurements.

In building a wind speed forecast model, the past records are used for training, hence how to organize them is critical to the performance of the forecast model. For a time series, it is necessary to take some of the previous records as inputs and the next one as the corresponding output. Here, same input patterns with different outputs may happen, especially when we employ a tremendous number of records collected during a long time interval. Different training pattern sets will lead to different forecast models. Wind speed tends to change differently for each season or month. Hence, some approaches develop independent models for each time period. However, the forecast of other period can be more accurate from time to time. In this regard, this paper develops a voting-based prediction scheme for wind speed change, targeting at Jeju City, Rep. of Korea, which is one of the smart grid leading cities in the world [8]. We build sequential, seasonal, monthly prediction models with ANNs and forecast the next hour speed by voting the outputs of 3 ANNs. This will avoid the unexpectedly inaccurate prediction occasionally found in a single model.

This paper is organized as follows: After outlining the whole paper in Section 1, Section 2 introduces related work on recent wind speed forecasts. Section 3 describes the main idea of this paper and shows the data processing framework. Section 4 evaluates the performance of the proposed scheme using the data not given to the training phase. Finally, Section 5 summarizes and concludes this paper with a brief introduction of future work.

\section{Related Work}

[9] proposes a spatio-temporal wind speed model for the short-term forecast of wind power generation. The authors begin with the assumption that the target station is surrounded by stations taking the same order AR (Auto Regressive) models. Then, a nonuniform multivariate AR method groups stations either having high or low crosscorrelation with the target station. Here, in AR models, the output variable of a system is well presented as a weighted linear combination of its own previous values. Usually, only a few of stations have a strong correlation with the target station and the correlation matrix must be block-sparse. Due to the nontrivial distribution of null spaces in the correlation matrix, there can be a lot of candidate solutions, however, only one is suitable under specific conditions. This scheme is applied to the speed records in the east coast area of the U.S., in which 57 stations are densely located. At the target station, blocksparse pattern appear in all of the other coefficient vectors. It also stresses its shorter computation time than other ANN-based approaches, achieving the reduction of forecast error in the target area by up to around $30 \%$.

[10] takes advantage of SVMs (Support Vector Machines) in wind speed forecasting. SVMs have been used not just in the weather forecast but also in pattern recognition, text categorization, and the like. Based on the concept of statistical learning, SVMrequires the consideration on feature space, loss functions, and kernel functions. The loss function maps an event to a real number, while the kernel functions are set of algorithms for pattern examination, including polynomial function, Gaussian basis function, and various splines. The input parameters of the SVM consist of the number of training data, permissible error bound, and kernel-related components such as limit, angle, and offset. The developed model is applied to a real wind speed record captured by National Renewable Energy Laboratory with a 5 minute interval. Here, it is indispensable to detect and correct bad data by means of any data mining process. According to their 
observation, the use of 4 data records reaches the optimal results with the employed kernel.

[11] presents a sophisticated deep-learning technique called PDBM (Predictive Deep Boltzmann Machine) for wind speed forecasting. A Boltzmann machine is a stochastic neural network capable of recognizing a probability distribution over given inputs. Here, the bottom-up connections correspond to the recognition relationships while the topdown connections to the weight of generative patterns. The proposed PDBM adds a predictive layer to the legacy DBM [12], incorporating a set of inference values. During its learning process, the pretraining phase finds good initial values for all predefined parameters, while the next fine-tuning phase runs the back propagation algorithm to keep refining them until the loss function reaches its minima. Then, it analyzes the higher level features abstracted from lower level ones of the wind speed records. This scheme is applied to the data set collected in southern China and the set has a 10 minute interval. The authors address that many issues are still open for PDBM including distributed and parallel learning algorithms.

[13] discovers the general trend of the incoming year by a data fusion scheme taking several neural networks. It employs the wind speed record for the past 23 years in the region of Mersing, Malaysia. For annual wind speed prediction, the average monthly speed of the coming year is forecasted based on the NARX (Nonlinear Auto Regressive network with eXogenous inputs). The output will be another time series and stored to make further feedback. Next, for the monthly prediction, interpolation functions process all the discrete data points to find a periodic behavior of the month-by-month wind speed change. Here, the neural network builds two feed-forward back propagation networks, each of which consists of two layers with 6 and 15 neurons. Finally, the general pattern of the next year is obtained after averaging and normalizing the hourly wind speed. This model suppresses the average forecast error to the $0.8 \mathrm{mps}$ (meter per second) boundary. Here, the authors have the model updated periodically to adapt to the forecast error deviation.

EMD (Empirical Mode Decomposition), originally developed for nonlinear time series problem solving, decomposes a time series into a collection of intrinsic mode functions and a residue, making it much easier to analyze divided ones. How to decompose the series is decided by local maxima, local minima, and zero-crossings. Such steps are quite empirical and thus this operation is essentially adaptive. EMD has many variants including ensemble EMD, complementary EMD, and complete EMD with adaptive noise. Each variant has its own characteristic. For example, ensemble EMD is a multiple trial process and its input series is basically a mixture of the original series and a finite Gaussian white noise. In [14], EMD and its variants are hybridized with SVR (Support Vector Regression) and ANN. To integrate SVR, the Lagrange multiplier method converts the optimization function to a quadratic optimization problem. The respective combinations are tested for the time series data from NDBC (National Data Buoy Center), where $70 \%$ is used for training. 


\section{Wind Speed Prediction Model}

\subsection{Data Analysis Framework}

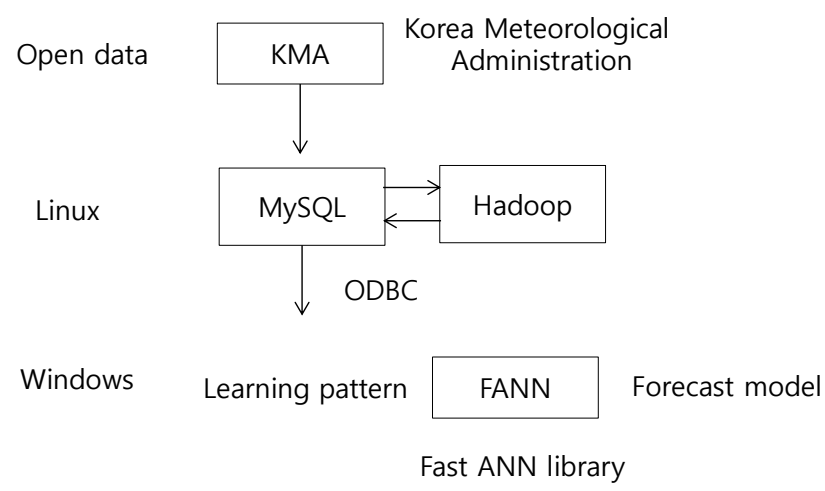

Figure 1. Hourly Trace

Figure 1 depicts the data processing architecture we are constructing. To begin with, KMA (Korea Meteorological Administration) publishes wind speed records along with other meteorological data, such as sunlight, precipitation, and the like, mainly on an hourly basis. After downloading the time series as a text file through the web interface ( $h t t p: / / w w w . k m a . g o . k r$ ), it is necessary to remove the garbage first. Then, the stream is converted to a series of SQL statements to insert into a database table. The table is defined in the MySQL database running on our Linux machine. The data analysis module retrieves necessary information through the MySQL communicators on its own platform. Here, the $\mathrm{R}$ statistics package supports a direct data connection to a remote MySQL server. Moreover, ODBC (Open DataBase Connectivity) allows an ordinary application to retrieve specific database fields and then process according to the analysis strategy in its workspace. Our work employs the FANN (Fast ANN) library which provides abundant ANN APIs to C language applications [15].

\subsection{ANN Voting Model}

Figure 2 shows the main idea of this paper, which builds 3 independent ANN categories. Basically, each ANN takes wind speeds for 5 previous hours as input to predict the next hour wind speed. That is, each ANN has 5 inputs and 1 output node. The number of hidden nodes is selected by trial and error. First, our scheme retrieves all the series records without any grouping from the database table and converts to training patterns. Here, the hourly speed records accumulated for 15 years are retrieved. The last year records are reserved for model evaluation. The training pattern file is fed to the FANN API functions to build $A N N 1$. The ANN does not differentiate the records collected in different seasons or months, but sequentially takes the training patterns. Next, the wind speed records of each season for each year are retrieved. Here, according to the season classification in the Rep. of Korea, 3 months from March belong to spring, and the next each 3 months to summer, fall, and winter, respectively. Hence, the blocks of 3 months are converted to training patterns. After all, we build 4 seasonal ANNs. The one selected according to the given day will be denoted by ANN2. Finally, our model builds 12 monthly ANNs, each of which is trained by the block of 1 month period. 


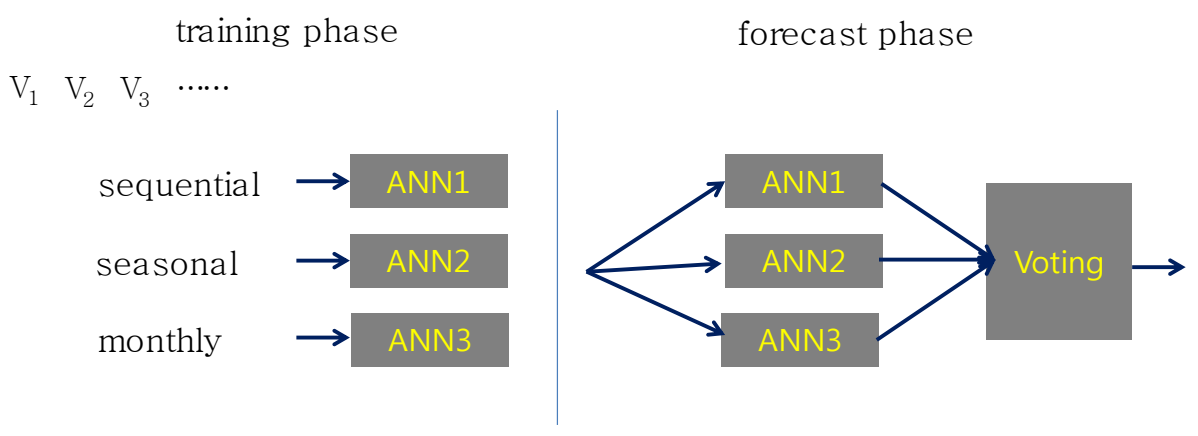

Figure 2. Hourly Trace

Each ANN has its own features. For example, the sequential ANN, or ANN1, can trace the overall change of the input patterns. As this model has the largest number of training patterns, it can trace the average behavior of the next hour wind speed for the given 5 previous speeds. Next, the ANN2 networks focuses on the season-by-season dependency on the previous records, so it can chase the season specific patterns. Likewise, the ANN3 networks take monthly dependency and have the smallest number of training patterns. To predict a next hour speed, it is necessary to identify the current month. According to this, we can select the corresponding ANNs. Then, the previous records are input to 3 ANNs simultaneously, so the 3 independent predictions are created. Our prediction module calculates the difference between each pair of them and the prediction farthest away from the other 2 will be excluded. The final prediction will be the average of the remaining 2 .

\section{Performance Measurement}

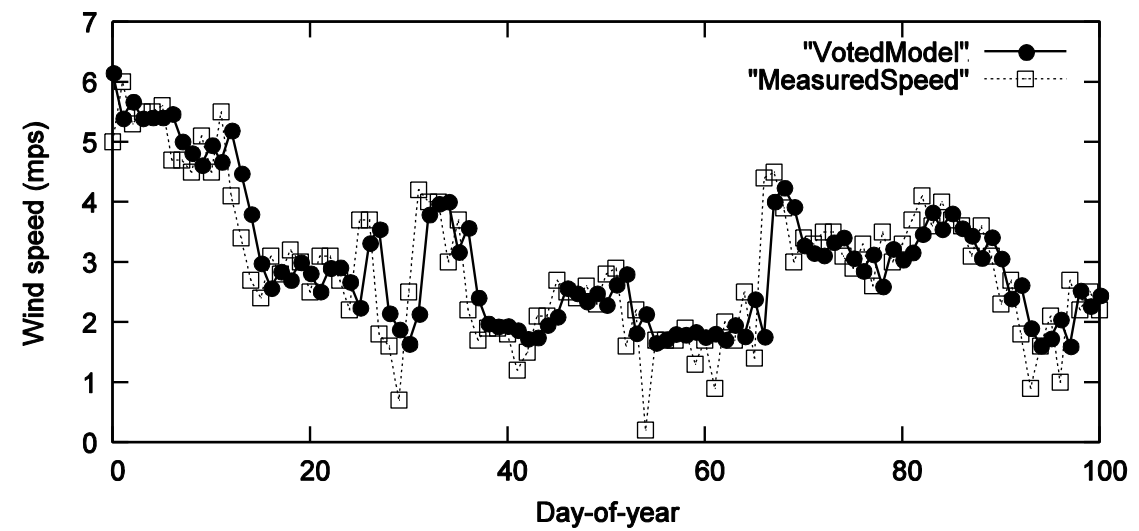

Figure 3. Hourly Trace For Predicted And Measured Wind Speeds

This section evaluates the accuracy of the voting-based wind speed forecast scheme in terms of general performance metrics in the time series modeling and forecasting such as RMSE (Root Mean Square Error), ME (Maximum error), MAE (Mean Absolute Error), and MAPE (Mean Absolute Percentage Error), comparing with pure sequential, monthly, seasonal models. As mentioned earlier, the hourly wind speed records from 1999 to 2013 are trained to build respective ANNs. Then, the speed records of 2014 are used for evaluation. To begin with, there are about $24 \times 365$ test inputs. Figure 3 plots the hourly change of measured (actual) speed and predicted speed, respectively. As full-year trace will be too dense to present in a single graph, Figure 3 plots the data only for the first 100 days. Just like other forecast models, the prediction error happens due to time lagging, and this makes the error quite large when the speed changes sharply. According to Figure 3 , our ANN predicts the hour-by-hour wind speed change, grabbing the general trends of the actual values. 
Next, Figure 4 compares the daily RMSE, which is calculated as in Equation (1).

$$
R M S E=\sqrt{\frac{1}{N} \sum_{h=1}^{N}\left(W_{h}^{m}-W_{h}^{p}\right)^{2}}
$$

, where is $W_{h}^{m}$ the actual measured speed and is $W_{n}^{p}$ the predicted speed at time $h$. The 24 predictions are analyzed against the actual values for each day. As shown in the figure, 4 curves have a similar pattern. Even if the proposed scheme, denoted by a solid line, has the lowest error level, he gap is not so significant. This is because sequential, monthly, and seasonal models predict the next hour wind speed quite similarly. Anyway, maximum daily RMSE for the proposed scheme is the smallest of the 4 by $0.11 \mathrm{mps}$ (meter per second). In addition, the seasonal model has the largest maximum daily RMSE, but it has the smallest number of days with error greater than $1.5 \mathrm{mps}$ and the largest number of days with error smaller than $0.5 \mathrm{mps}$. The proposed scheme oscillates between 3 models, outperforming on average and showing stable error characteristics.

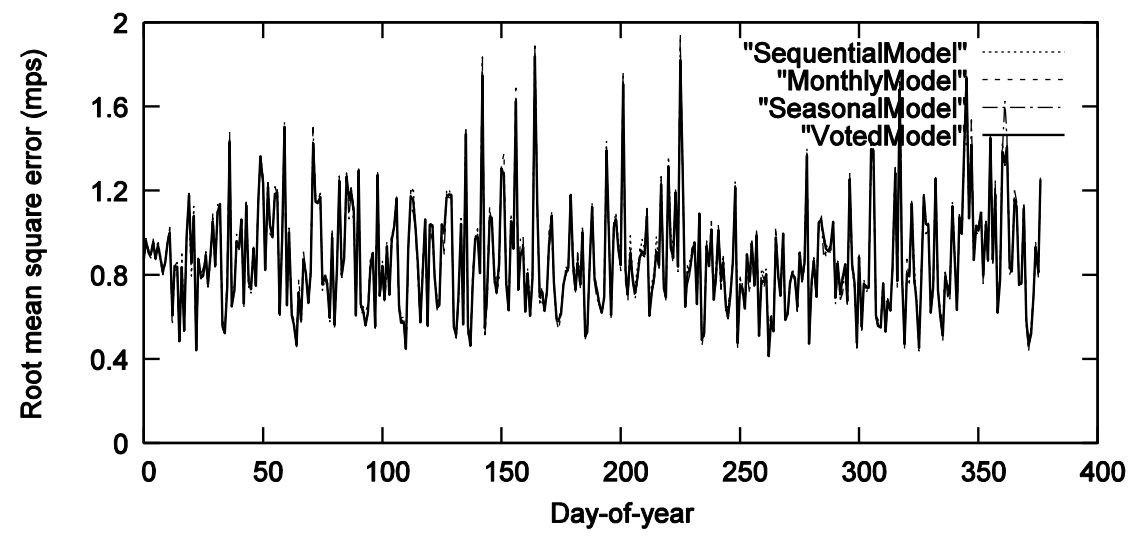

Figure 4. Daily Root Mean Square Error

Now, Figure 5 plots the maximum prediction error for each day, and this error is calculated as shown in Equation (2).

$$
M E=\max \left(\left|W_{h}^{m}-W_{h}^{p}\right|\right)
$$

Here again, $h$ can be one of 24 hours. The daily maximum reaches near $6 \mathrm{mps}$ from time to time, but mainly remains below $2.5 \mathrm{mps}$. Even in the case 23 predictions are accurate but one has a significant error, the daily maximum will be the same as the worst error. On those days when the wind speed changes abruptly, the maximum error will be larger. The prediction model seems to chase the actual pattern change usually with small time lag. Particularly, if the speed changes from $0 \mathrm{mps}$ to 6 or higher in an hour and the model catches up 1 hour later, the error size will be 6 or higher. Nevertheless, the daily maximum remains around $2 \mathrm{mps}$ even on those days which are highly likely to have experienced a significant change from their closeness to large-error days. Actually, in terms of the maximum daily error, the sequential model shows the smallest error of 5.34 mps, while the proposed scheme shows $5.79 \mathrm{mps}$. The number of days whose maximum error exceeds 5.0 mps is smallest on the seasonal model. 


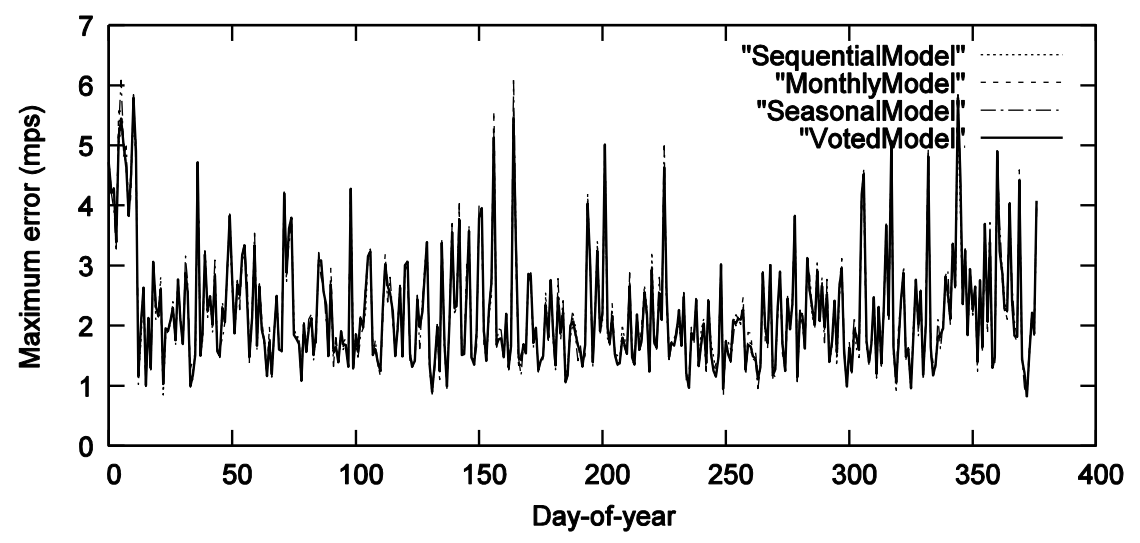

Figure 5. Daily Maximum Error

Figure 6 shows the daily maximum absolute error, and it will be calculated as shown in Equation (3).

$$
M A E={ }^{\frac{1}{N}} \sum_{h=1}^{N}\left|W_{h}^{m}-W_{h}^{p}\right|
$$

Unlike the daily maximum error, the average error shows the general accuracyofr the prediction. The voting scheme usually comes at the lowest place. Sometimes, the most and second most erroneous predictions are close and win the vote, counting out the accurate one. In this case, our scheme can have significant errors. Anyway, in terms of the maximum of daily mean error, the vote-based scheme outperforms the others by 0.01 mps. For the number of days having errors larger than $1.0 \mathrm{mps}$, only the seasonal model works poorer than the other three by $17.3 \%$. In addition, for the number of days having errors of $0.5 \mathrm{mps}$ or less, the proposed scheme outperforms the others by from 4.5 to 18.1 $\%$.

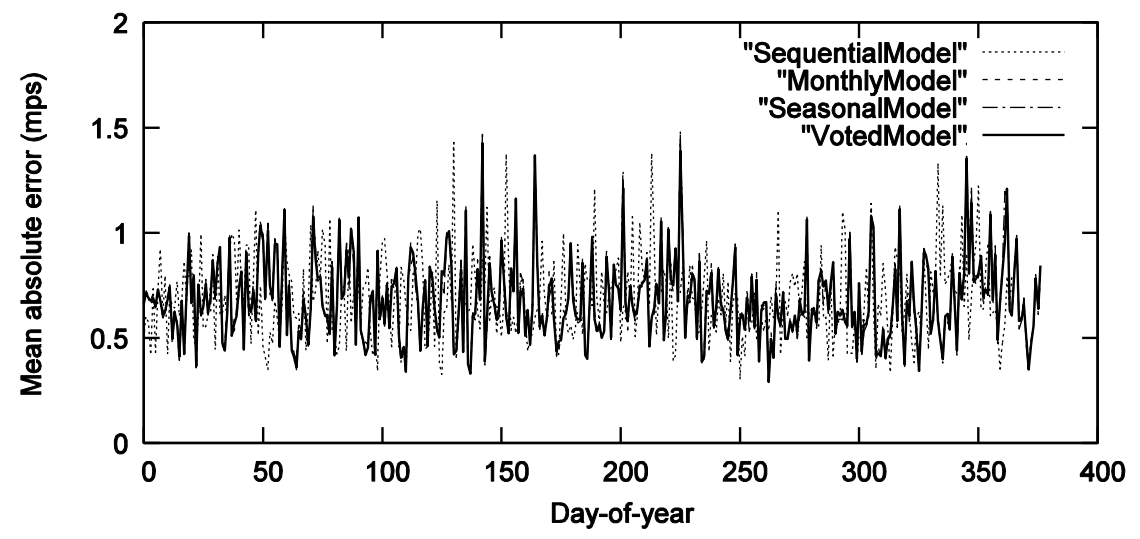

Figure 6. Daily Mean Absolute Error (4).

Figure 7 plots the mean average percentage error, which is calculated as shown in Eq.

$$
\text { MAPE }=\frac{100 \%}{N} \sum_{h=1}^{N}\left|\frac{W_{h}^{m}-W_{h}^{p}}{W_{h}^{m}}\right|
$$

This metric measures how close a prediction is to its real value. As can be seen in Equation (4), the measured value comes in the denominator. Hence, ${ }^{W_{h}^{m} \text { if approaches }}$ zero, even a small error leads to a significant percentage error. Moreover, those hours having the wind speed of 0 will be eliminated in computing the percentage error. While 
the hours having a $100 \%$ or larger percentage error appear in the graph, in most days, the error size remains below $40 \%$. In summer season, most days are not windy but the wind speed changes unpredictably in a short time according to rainstorms and the like. Hence, the percentage error goes quite high during this season. Even though the maximum percentage error seems a little bit meaningless, the error of around $150 \%$ happens in all 4 schemes.

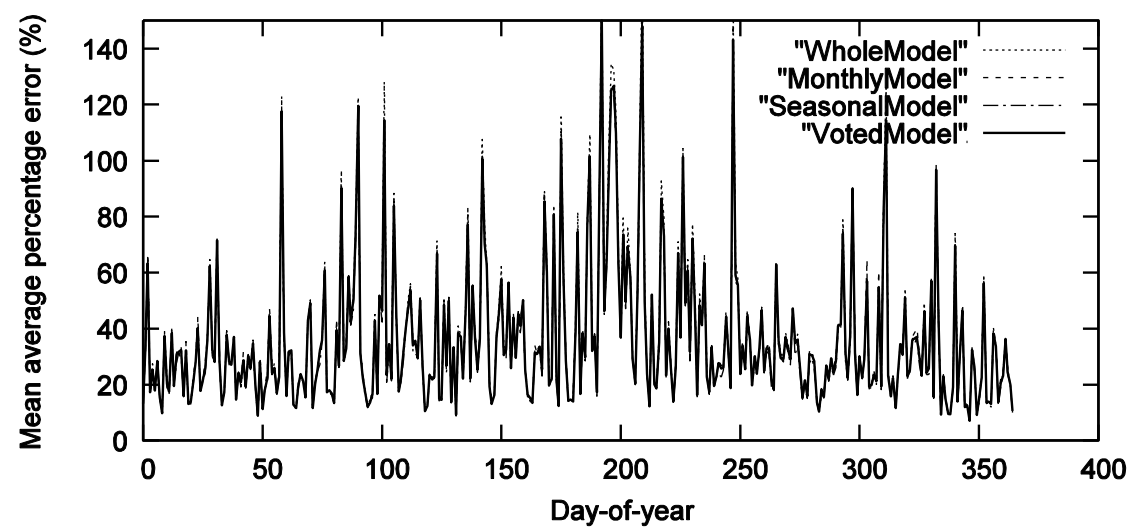

Figure 7. Daily Mean Average Percentage Error

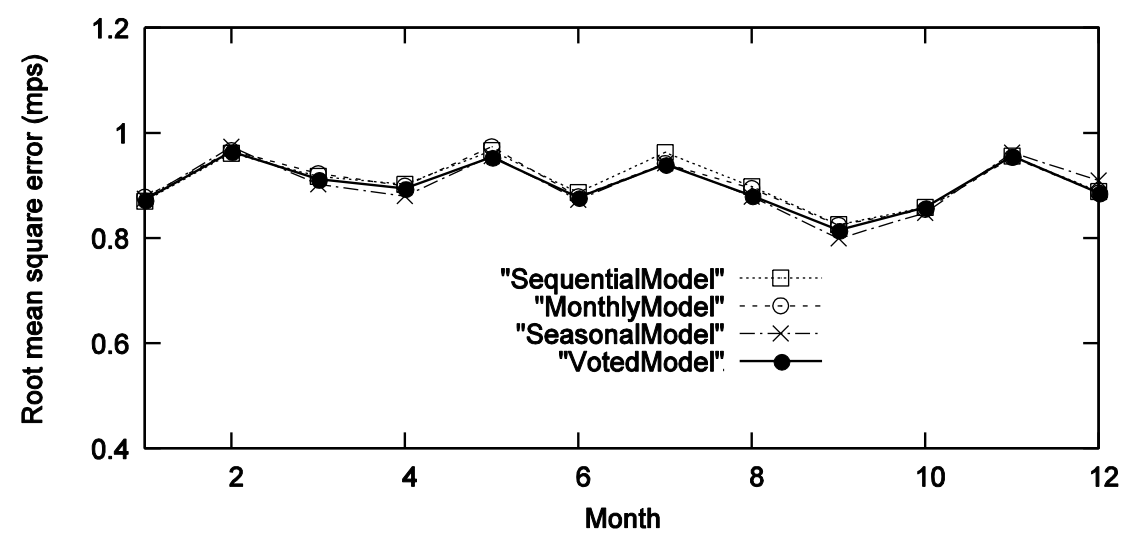

Figure 8. Monthly Root Mean Square Error

Now, we turn our focus to monthly statistics. Each metric is calculated on a monthly basis. First, Figure 8 shows the monthly root mean square error. Contrary to the daily statistics, the curves are quite smooth. The monthly error falls into the range from 0.81 to $0.96 \mathrm{mps}$ range. Actually, the root mean square error for each model, that is, the yearly statistics is almost same. In the proposed scheme, the gap between the maximum and minimum errors becomes smaller. In addition, the vote-based scheme avoids the worst case mis-prediction. Miscellaneously, the seasonal prediction has the maximum and minimum monthly errors.

Figure 9 shows the monthly maximum error. As the worst prediction of the month is selected, the error curves go higher for all cases. While the proposed scheme doesn't have the smallest error in each month, it doesn't have sharp peaks even in the highly unpredictable summer season. As for seasonal model, the maximum error goes higher. It means that seasonal grouping is a little bit meaningless or inappropriate. However, it shows the smallest error from time to time. A more sophisticated complementary method could overcome this shortcoming. 


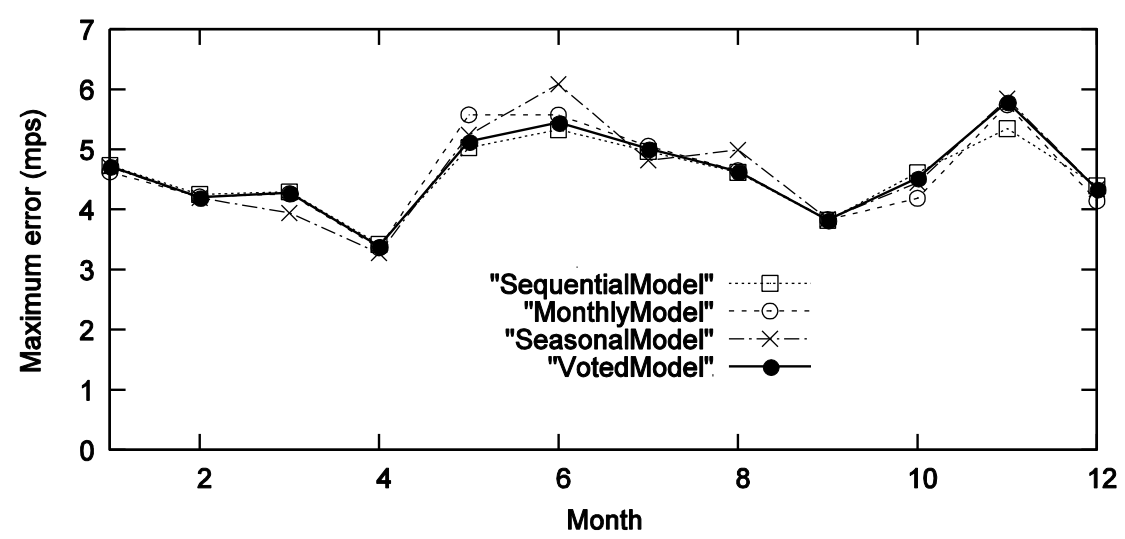

Figure 9. Monthly Maximum Error

Figure 10 plots the monthly absolute error. Here again, the gap between the maximum and minimum values is smallest in the vote-based scheme. Just like the hourly trace, the error of the proposed scheme tends to stick to the mean of the lowest two. Except quite a few cases two largest errors block out the most accurate prediction, the voting process can reduce the effect of the penetration of errors.

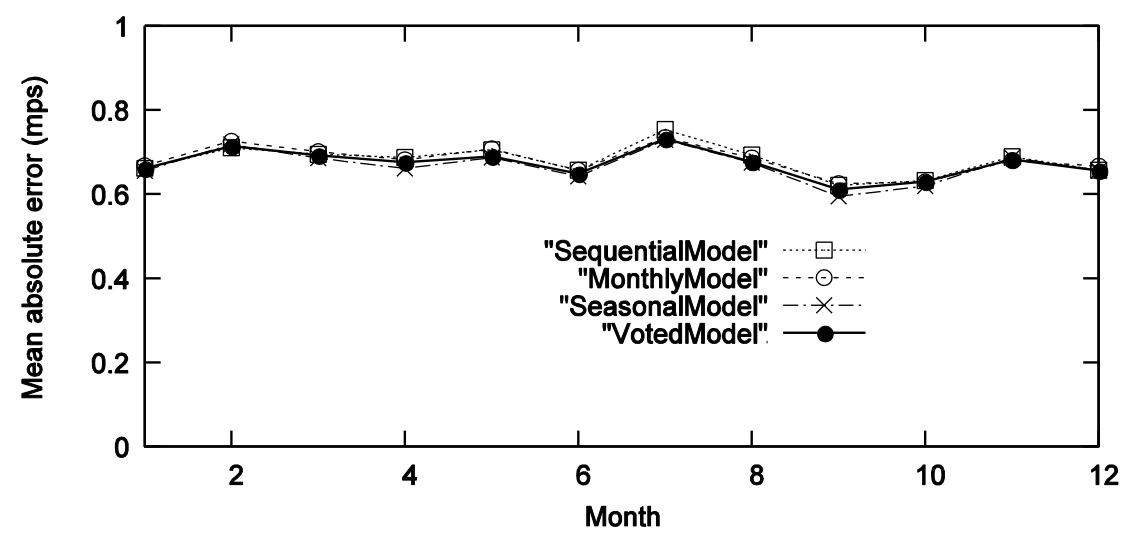

Figure 10. Monthly Maximum Absolute Error

Finally, Figure 11 plots the monthly mean average percentage error. The error goes very high during the summer season, specifically in July, which corresponds to the monsoon season and faces typhoons. 4 curves behave quite similarly to the case of Figure 10.

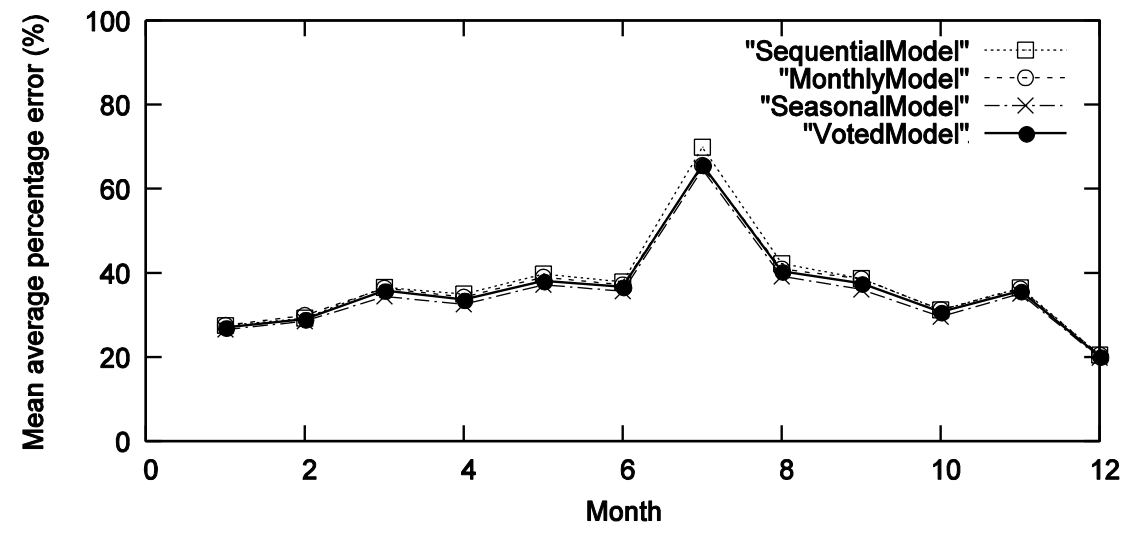

Figure 11. Monthly Mean Average Percentage Error 


\section{Conclusions}

Smart grids are trying to integrate more and more renewable energies to reduce air pollutions and enhance energy efficiency. To overcome the intermittency of the wind energy, not only battery technologies but also the future wind speed prediction is important for efficient planning of power generating planning and facility management. In this paper, we have designed and implemented a vote-based wind speed forecast scheme for Jeju city, which has outstanding wind power generation potential. The hourby-hour wind speed records from 2004 to 2013 are downloaded from KMA via its open data web interface and stored in our own database. Our analyzer classifies and converts the records to training patterns according to the month or season to which the timestamp of a record belongs to. After the training phase, 1 ANN for sequential modeling, 4 ANNs for seasonal modeling, and 12 ANNs for monthly modeling are created. To predict the next hour speed, the speed records of the previous 5 hours are simultaneously fed to each model first. Then, the voting process picks and averages the two predictions having the best proximity out of 3 .

The evaluation process compares the actual speed and the predicted speed for the speed records of 2014 which is not used for training. The experiment has yielded daily and monthly statistics on RMSE, ME, MAE, and MAPE, respectively. It finds out that maximum daily root mean square error for the proposed scheme is the smallest of the 4 by $0.11 \mathrm{mps}$, while its prediction has the smallest error out of 4 mentioned models in most cases. Moreover, the vote-based scheme avoids the worst case mis-prediction, which can be observed in a single prediction model.

As future work, we are planning to incorporate interdependent time series streams created from a variety of smart grid entities such as electric vehicles, charging facilities, microgrids, distributed generators, and the like, to construct an intelligent smart grid city and find a promising business model based on the big data analysis.

\section{References}

[1] C. Chakraborty, H. Ho-Ching Iu, and D. Dah-Chuan Lu, "Power converters, control, and energy management for distributed generation", IEEE Transactions on Industrial Electronics, vol. 62, no. 7 , (2015), pp. 4466 - 4470.

[2] U. Annakkage, D. Jacobson, and D. Muthumum, "Method for studying and mitigating the effects of wind variability on frequency regulation", CIGRE/IEEE PES Join Symposium on Integration of WideScale Renewable Resources into the Power Delivery System, (2009).

[3] S. Du and C. Zuo, "Driving ranges prediction of pure electric vehicle with dual energy storage system based on BP neural network", International Journal of u- and e- Service, Science and Technology, vol. 8, no.3, (2015), pp. 273-286.

[4] R. Jursa, "Variable selection for wind power prediction using particle swarm optimization", 9th Annual Conference on Genetic and Evolutionary Computation, (2007), pp. 2059-2065.

[5] N. Abdel-Karim, M. Small, and M. Ilic, "Short term wind speed prediction by finite and infinite impulse response filters: A state space model representation using discrete Markov process", IEEE Bucharest Power Tech Conference, (2009).

[6] C. Wang, Z. Lu, and Y. Qiao, "Modeling of wind pattern and its application in wind speed forecasting", International Conference on Sustainable Power Generation and Supply, (2009), pp. 1-6.

[7] K. Methaprayoon, C. Yingvivatanapong, W. Lee, and J. Liao, "An integration of ANN wind power estimation into unit commitment considering the forecasting uncertainty", IEEE Transactions on Industry Applications, vol. 43, (2007), pp. 1441-1448.

[8] J. Lee, G. Park, Y. Kim, E. Kim, and I. Lee, "Wind speed modeling based on artificial neural networks for Jeju area", International Journal of Control and Automation, vol. 5, no. 2, (2012), pp. 73-80.

[9] B. Sanandaji, A. Tascikaraoglu, K. Poolla and P. Varaiya, "Low-dimensional models in spatiotemporal wind speed forecasting", American Control Conference, (2015), pp. 4485-4490.

[10] T. Pinto, S. Ramos, R. Sousa and Z. Vale, "Short-term wind speed forecasting using support vector machines", IEEE Symposium on Computational Intelligence in Dynamic and Uncertain Environments, (2014), pp. 40-46.

[11] C. Zhang, C. Chen, M. Gan and L. Chen, "Predictive deep Boltzmann machine for multiperiod wind speed forecasting", International Journal of Control and Automation, IEEE Transactions on Sustainable Energy, vol. 6, no. 4, (2015), pp. 1416-1425. 
[12] R. Salakhutdinov and G. Hinton, “An efficient learning procedure for deep Boltzmann machine”, Neural Computing, vol. 14, no. 8, (2012), pp. 1967-2006.

[13] H. Azad, S. Mekhilef and V. Ganapathy, "Long-term wind speed forecasting and general pattern recognition using neural networks", IEEE Transactions on Sustainable Energy, vol. 5, no. 2, (2014), pp. 546-553.

[14] Y. Ren, P. Suganthan, and N. Srikanth, "A comparative study of empirical mode decomposition-based short-term wind speed forecasting methods", IEEE Transactions on Sustainable Energy, vol. 6, no. 1, (2015), pp. 236-244.

[15] S. Nissen, Neural Network Made Simple, Software 2.0, (2005). 
International Journal of Control and Automation Vol. 9 No. 5 (2016) 